\title{
$\beta 1$ Integrins Mediate Attachment of Mesenchymal Stem Cells to Cartilage Lesions
}

Daniela Zwolanek, ${ }^{1}$ Magdalena Flicker, ${ }^{1}$ Elisabeth Kirstätter, ${ }^{1}$ Frank Zaucke, ${ }^{2}$ Gerjo J.V.M. van Osch, ${ }^{3}$ and Reinhold G. Erben ${ }^{1, *}$

\begin{abstract}
Mesenchymal stem cells (MSC) may have great potential for cell-based therapies of osteoarthritis. However, after injection in the joint, only few cells adhere to defective articular cartilage and contribute to cartilage regeneration. Little is known about the molecular mechanisms of MSC attachment to defective articular cartilage. Here, we developed an ex vivo attachment system, using rat osteochondral explants with artificially created fullthickness cartilage defects in combination with genetically labeled MSC isolated from bone marrow of human placental alkaline phosphatase transgenic rats. Binding of MSC to full-thickness cartilage lesions was improved by serum, but not hyaluronic acid, and was dependent on the presence of divalent cations. Additional in vitro tests showed that rat MSC attach, in a divalent cation-dependent manner, to collagen I, collagen II, and fibronectin, but not to collagen XXII or cartilage oligomeric matrix protein (COMP). RGD peptides partially blocked the adhesion of MSC to fibronectin in vitro and to cartilage lesions ex vivo. Furthermore, the attachment of MSC to collagen I and II in vitro and to cartilage lesions ex vivo was almost completely abolished in the presence of a $\beta 1$ integrin blocking antibody. In conclusion, our data suggest that attachment of MSC to ex vivo full-thickness cartilage lesions is almost entirely $\beta 1$ integrin-mediated, whereby both RGD- and collagen-binding integrins are involved. These findings suggest a key role of integrins during MSC attachment to defective cartilage and may pave the way for improved MSC-based therapies in the future.
\end{abstract}

Key words: biochemistry; cellular biology; extracellular matrix; stem cells

\section{Introduction}

Cell therapies or cell-based gene therapies with adult multipotent mesenchymal stem cells (MSC) may have a large impact on the treatment of many diseases of various organ systems in the future. ${ }^{1}$ One of the typical diseases in which MSC-based therapies may hold great promise is osteoarthritis (OA). OA is the most common age-related form of arthritis with prevalence for the knee and hip joint. The initiation of the disease is triggered by genetic disposition, single major trauma, a series of microtraumas, or strenuous exercise. ${ }^{2}$ The few differentiated chondrocytes within the articular cartilage have only minimal regeneration potential, ${ }^{3}$ lead- ing to a poor prognosis for patients suffering from OA. ${ }^{4}$ Murphy et al. demonstrated that the intra-articular injection of autologous bone marrow-derived MSC in a goat model of OA stimulated the regeneration of meniscal tissue and retarded progressive joint destruction. ${ }^{5}$ Since then, other studies investigating cartilage regeneration using MSC also reported successful and promising results in various model systems. ${ }^{6-10}$ However, only a small fraction of injected cells adhere to damaged cartilage and therefore contribute to tissue regeneration. ${ }^{11}$ In addition, it is currently a matter of debate whether the beneficial effects of MSC therapy is due to a progenitor or a nonprogenitor function of

\footnotetext{
${ }^{1}$ Department of Biomedical Sciences, University of Veterinary Medicine, Vienna, Austria.

${ }^{2}$ Center for Biochemistry and Cologne Center for Musculoskeletal Biomechanics, Medical Faculty, University of Cologne, Cologne, Germany.

${ }^{3}$ Department of Orthopaedics and Otorhinolaryngology, Erasmus MC, University Medical Center Rotterdam, Rotterdam, The Netherlands.

*Address correspondence to: Reinhold G. Erben, MD, DVM, Department of Biomedical Sciences, University of Veterinary Medicine Vienna, Veterinaerplatz 1, A-1210 Vienna, Austria, E-mail: reinhold.erben@vetmeduni.ac.at

(c) Daniela Zwolanek et al. 2015; Published by Mary Ann Liebert, Inc. This Open Access article is distributed under the terms of the Creative Commons License (http://creativecommons.org/licenses/by/4.0), which permits unrestricted use, distribution, and reproduction in any medium, provided the original work is properly credited.
} 
the injected cells. ${ }^{12}$ Therefore, the clinical potential of MSC-based therapy of OA is currently unclear.

MSC are a cell population of undifferentiated cells isolated from adult bone marrow or other tissues with the capacity to differentiate into mesodermal lineages such as bone, cartilage, muscle, or fat tissue. They can be isolated and expanded with low effort and can be differentiated into multiple cell lineages under certain culture conditions. Cultured MSC are positive for the surface markers CD44, CD73, CD90, CD105, CD106, $\mathrm{CD} 120 \mathrm{a}$, and CD124. ${ }^{13}$ Beside those, they also express various integrin subunits such as $\alpha 1, \alpha 2, \alpha 3, \alpha 4, \alpha 5, \alpha 6$, $\alpha 7, \alpha 10, \alpha \mathrm{V}, \alpha \mathrm{IIb}, \beta 1, \beta 3, \beta 4$, and $\beta 5{ }^{13-15}$ Integrins are thought to play a pivotal role in adhesion of MSC to extracellular matrix (ECM) proteins and biocompatible polymers. ${ }^{16-18}$ The noncovalent binding of an $\alpha$ - and a $\beta$-subunit builds a full integrin, which is activated, and stretched in length, by binding to intracellular or extracellular proteins that drive the signaling to the ECM or to the cell. ${ }^{19-21}$ The integrin family consists of five different groups depending on their ligand binding. For example, collagen-binding integrins prefer the binding to the collagenous triple helix, whereas RGD-binding integrins, the largest subgroup, bind exclusively the amino acid motif Arg-Gly-Asp. ${ }^{22}$ Integrins mediate processes such as adhesion, migration, proliferation, cytoskeleton organization, and survival. ${ }^{20}$

In order to function as progenitors for cartilage regeneration intra-articularly injected MSC need to attach to articular cartilage lesions. Articular cartilage can be divided into distinct zones depending on the organization and shape of the embedded chondrocytes. The superficial zone, the outermost zone of the articular cartilage harbors only few differentiated chondrocytes with a flattened cell shape that are organized in parallel and embedded in a tight ECM. ${ }^{3}$ The cartilage ECM consists of high amounts of collagens and proteoglycans that build large aggregates embedded into the collagen network. ${ }^{23}$ The predominant collagen in articular cartilage is collagen II that forms fibrils with integrated collagen IX and XI. ${ }^{24}$ Noncollagenous proteins such as COMP (cartilage oligomeric matrix protein), matrilin-3, biglycan, decorin, and aggrecan, as well as the glycosaminoglycan hyaluronan, and other factors are needed to interconnect the different networks, thereby strengthening the ECM in articular cartilage. ${ }^{25}$ Cartilage defects result in the degradation of the ECM followed by inflammatory processes, apoptosis of chondrocytes, and subsequent loss of articular cartilage and sclerosis of the subchondral bone in combination with osteophyte formation, ultimately leading to OA.

Currently, only little is known about the molecular mechanisms involved in MSC adhesion to cartilage lesions. Here, we set out to characterize the attachment of rat MSC to full-thickness cartilage lesions and to ECM components in more detail, using a combination of ex vivo and in vitro systems. To this end, we first developed an ex vivo attachment system, using rat osteochondral explants with full-thickness cartilage defects in combination with genetically labeled, bone marrow-derived rat MSC. To identify the attached MSC on the tibial explants, we used human placental alkaline phosphatase (hPLAP) expressing MSC, which harbor the same characteristics as wild-type cells and are easy to track by histochemical staining. ${ }^{26,27}$ hPLAP is a heat-stable marker enzyme that retains its enzymatic activity after paraffin and plastic embedding. ${ }^{26} \mathrm{Next}$, we investigated the effect of adjuvants such as hyaluronic acid, serum, plasma, and divalent cations on MSC attachment to cartilage lesions. Finally, we analyzed the attachment of MSC to individual ECM proteins in vitro. MSC were able to bind to bone and cartilage ECM proteins via $\beta 1$ integrins of the collagenbinding and RGD-binding integrin family. These findings suggest a key role of integrins during MSC attachment to defective cartilage and may pave the way for improved cell-based therapies with MSC in the future.

\section{Materials and Methods}

Animals

All animal experiments were approved by the ethics committees of the University of Veterinary Medicine and of the local government authorities. Wild-type and hemizygous female R26-hPLAP-tg inbred Fischer 344 rats originally generated by Kisseberth and coworkers, ${ }^{28}$ ubiquitously expressing the marker gene hPLAP, were genotyped by enzymatic histochemistry using a drop of tail blood. ${ }^{28,29}$ Rats were housed in groups of $2-6$ at $24^{\circ} \mathrm{C}$ and a $12 \mathrm{~h} / 12 \mathrm{~h}$ light/dark cycle with free access to tap water and a standard rat diet (Ssniff).

\section{MSC isolation}

Bone marrow-derived MSC were isolated from wildtype and hPLAP-tg female rats at the age of 3-6 weeks according to the protocol described by Balmayor et al. $^{27}$ In brief, long bones (femur, tibia, and humerus) were defleshed, cut at the epiphysis, and subsequently digested with $2.5 \mathrm{mg} / \mathrm{mL}$ collagenase type II (Invitrogen) 
for $2 \mathrm{~h}$. The bone marrow was flushed out with complete medium containing Eagle's minimum essential medium (MEM; PAA, GE Healthcare), 10\% fetal bovine serum (FBS; PAA, GE Healthcare), and 1\% penicillin/ streptomycin (PAA, GE Healthcare), and centrifuged. With the help of a Ficoll gradient centrifugation (Ficoll-Paque; GE Healthcare) the mononuclear cell fraction was collected, washed, and seeded in cell culture flasks (TPP; Trasadingen). After $24 \mathrm{~h}$, the medium was changed and the cells were grown until $80 \%$ confluence. Cells were passaged up to passage 4. During cultivation, the medium was changed twice a week and cells were kept at $37^{\circ} \mathrm{C}$ with $5 \% \mathrm{CO}_{2}$ and $3 \% \mathrm{O}_{2}$.

Ex vivo attachment assay with osteochondral explants Tibiae from wild-type rats at the age of 5-7 months were isolated, cleaned from surrounding soft tissue without damaging the articular cartilage, and kept in PBS (without $\mathrm{Ca}^{2+}$ and $\mathrm{Mg}^{2+}$; PAA, GE Healthcare). Full-thickness defects with a diameter of $1 \mathrm{~mm}$ were created on the lateral and medial compartment of the tibia's articular cartilage with the help of a biopsy punch. Some tibia plateaus were left intact as a control. The tibia plateau was broken at the epiphysis, and the resulting osteochondral explants were separated into the medial and lateral compartment with a scalpel. Each explant was placed into a well of a 96-well plate (TPP; Trasadingen), washed, and kept in complete medium overnight at $37^{\circ} \mathrm{C}, 5 \% \mathrm{CO}_{2}$, and $3 \% \mathrm{O}_{2}$. The explants were placed into a new 96-well plate and washed 30-60 min with PBS. Single-cell suspensions of $1 \times 10^{4}$ hPLAP-tg MSC per explant at passage 1-4 were incubated on upright-positioned explants for 55 min unless otherwise indicated at $37^{\circ} \mathrm{C}, 5 \% \mathrm{CO}_{2}$, and $3 \% \mathrm{O}_{2}$ in physiological saline (Fresenius) alone or supplemented with rat serum (native, isolated whole blood of wildtype rats) or bovine serum (FBS; PAA, GE Healthcare), plasma (native, isolated from whole blood of wild-type rats, using EDTA as anticoagulant), bone marrow plasma (native, isolated from wild-type rats), hyaluronic acid (sodium salt from Streptococcus equi; Sigma), divalent cations $\left(\mathrm{MgCl}_{2}, \mathrm{CaCl}_{2}, \mathrm{ZnCl}_{2}\right.$, and $\mathrm{MnCl}_{2}$; Sigma), or $10 \mathrm{mM}$ EDTA (Sigma).

For blocking and competition assays, saline supplemented with $50 \%$ serum and $\beta 1$ integrin blocking antibody (clone $\mathrm{Ha} 2 / 5$; $\mathrm{BD}$ Biosciences), hamster IgM $\lambda 1$ isotype control (BD Biosciences), linear RGD peptide (GRGDNP), or linear RGD control peptide (GRADSP) (both from Enzo Life Sciences) were used. The explants were washed twice with PBS to remove nonadherent cells. Adherent cells were fixed with icecold acetone-methanol $(30: 70 \mathrm{v} / \mathrm{v})$ for $3 \mathrm{~min}$ at $-20^{\circ} \mathrm{C}$. After a washing step, the explants were placed in $1.5 \mathrm{~mL}$ tubes containing $1 \mathrm{~mL}$ PBS and incubated at $72^{\circ} \mathrm{C}$ and $200 \mathrm{rpm}$ for $90 \mathrm{~min}$ to inactivate endogenous alkaline phosphatases. Subsequently, explants with attached cells were stained for heat-stable hPLAP by using TNM buffer $(100 \mathrm{mM}$ Tris- $\mathrm{HCl}, \mathrm{pH} 9.5$, $100 \mathrm{mM} \mathrm{NaCl}, 5 \mathrm{mM} \mathrm{MgCl}_{2}$ ) containing $0.175 \mathrm{mg} /$ $\mathrm{mL}$ 3-bromo-4-chloro-3-indolyl phosphate (BCIP; Sigma) and $0.45 \mathrm{ng} / \mathrm{mL}$ nitrotetrazolium blue chloride (NBT; Sigma) for $3 \mathrm{~h}$ at room temperature (RT). The staining solution was replaced by PBS, and the explants were photographed under a stereomicroscope (Stemi SV6; Zeiss).

The obtained pictures were formatted to a size of $35 \mathrm{~cm} \times 35 \mathrm{~cm}$ and a cutout of $10 \mathrm{~cm} \times 15 \mathrm{~cm}$ with the defect in its center taken. The cutouts were analyzed with ImageJ software, and the area of attached and stained MSC was expressed as percent of the defect area. All measurements were done in triplicate with explants from three different animals per group, and repeated independently at least three times on different days with different MSC batches. Identically treated cell-free explants were used as additional negative controls.

\section{Histochemistry of cryosections}

hPLAP-stained and quantified osteochondral explants were fixed in $70 \%$ ethanol for $48 \mathrm{~h}$ and decalcified with $0.5 \mathrm{M}$ EDTA, $\mathrm{pH} 8.0$, for 4 weeks at $4^{\circ} \mathrm{C}$. Thereafter, the explants were embedded in TissueTek (Sakura Finetek), shock-frozen in liquid $\mathrm{N}_{2}$, and kept at $-80^{\circ} \mathrm{C}$ until sectioning. Eleven-micrometer-thick cryosections were transferred to 3-aminopropyltriethoxy-silane (APES; Sigma)-pretreated slides. For hPLAP staining, sections were fixed with acetonemethanol $(30: 70 \mathrm{v} / \mathrm{v})$ for $3 \mathrm{~min}$ at $-20^{\circ} \mathrm{C}$. As the decalcification procedure inactivates the hPLAP marker gene, reactivation had to be performed as described earlier. ${ }^{30}$ Therefore, all alkaline phosphatases were reactivated with $1 \% \mathrm{MgCl}_{2}$ in $100 \mathrm{mM}$ Tris-maleate buffer, $\mathrm{pH}$ 9.2, overnight in darkness. To inactivate endogenous alkaline phosphatases, sections were subsequently incubated at $65^{\circ} \mathrm{C}$ for $35 \mathrm{~min}$. The staining was carried out as described in detail before. ${ }^{29}$ Nuclear fast red (Sigma) was used as counterstain. For Alcian blue-PAS (periodic acid-Schiff) staining, a PAS staining kit (Merck) was used according to manufacturer's instructions after sections were fixed with $4 \%$ PFA 
(paraformaldehyde) for $5 \mathrm{~min}$ at RT. Pictures of the stained sections were taken with a microscope (Axioskop 2; Zeiss).

Immunofluorescence staining of cryosections

Anti-integrin immunofluorescence staining was performed on cryosections of hPLAP-prestained osteochondral explants as described previously. ${ }^{31,32}$ In brief, sections were fixed with ice-cold acetone for 2 min at $-20^{\circ} \mathrm{C}$ and blocked with $5 \%$ normal goat serum (Vector Laboratories) in PBS supplemented with $0.2 \%$ Tween-20 for $30 \mathrm{~min}$ at RT. Cryosections were stained overnight at $4^{\circ} \mathrm{C}$ for collagen I (rabbit polyclonal; Rockland Immunochemicals), collagen II (rabbit polyclonal; Rockland Immunochemicals), $\alpha 1$ integrin (hamster clone Ha31/8; BD Biosciences), $\alpha 2$ integrin (mix of three rat clones; Emfret), and $\alpha 5$ integrin (rabbit polyclonal; Santa Cruz Biotechnology), or a combination of $\alpha 2$ integrin and collagen I or collagen II for co-immunofluorescence staining, followed by incubation with FITC-conjugated goat anti-rabbit (Sigma), Alexa 488-conjugated goat anti-rat (Molecular Probes), Texas Red-conjugated goat anti-hamster (Vector Laboratories), and Cy3-conjugated goat anti-rabbit (Jackson ImmunoResearch), either single or in mixture, for $1 \mathrm{~h}$ at RT. Stained sections were analyzed and pictures were taken with a microscope (Axioskop 2; Zeiss).

In vitro cell attachment assay

In vitro cell attachment assays were carried out according to Eble et al. ${ }^{33}$ In brief, the following proteins were immobilized overnight at $4^{\circ} \mathrm{C}$ onto 96-well plates (Nunc): collagen I (bovine; Sigma), collagen II (rat, native extracted from trachea described by Dodge and Poole ${ }^{34}$ ), collagen XXII (human, gift of Manuel Koch, University of Cologne ${ }^{32}$ ), fibronectin (human; Sigma), COMP (rat, recombinant protein according to Hansen et al. $^{35}$ ), and bovine serum albumin (BSA; Sigma). Free binding sites were blocked with 1\% BSA in PBS for 3$4 \mathrm{~h}$ at $4^{\circ} \mathrm{C}$. Single-cell suspensions of wild-type or hPLAP-tg MSC at passage 1-4 were incubated for $55 \mathrm{~min}$ at $37^{\circ} \mathrm{C}, 5 \% \mathrm{CO}_{2}$, and $3 \% \mathrm{O}_{2}$ in physiological saline, or MEM alone or in combination with different concentrations of divalent cations $\left(\mathrm{MgCl}_{2}, \mathrm{CaCl}_{2}\right.$, $\mathrm{ZnCl}_{2}$, and $\mathrm{MnCl}_{2}$ ), $1 \mathrm{mM} \mathrm{MnCl}_{2}$ and $2 \mathrm{mM} \mathrm{MgCl}$, or $10 \mathrm{mM}$ EDTA at an average density of $6 \times 10^{4}$ cells/mL. For blocking and competition assays, MEM supplemented with $1 \mathrm{mM} \mathrm{MnCl} 2$ and $2 \mathrm{mM} \mathrm{MgCl}_{2}$ and $\beta 1$ integrin blocking antibody, hamster IgM $\lambda 1$ isotype control, RGD peptide, and RGD control pep- tide were used. Nonadherent cells were removed by washing with PBS and adherent cells fixed with $1 \%$ glutaraldehyde (Sigma) in PBS for $15 \mathrm{~min}$ at RT. After staining with $0.1 \%(\mathrm{w} / \mathrm{v})$, crystal violet cells were lysed with $0.2 \%$ Triton X-100 (Sigma). The amount of released dye was determined by measuring the absorption at $570 \mathrm{~nm}$ using a microplate reader (EnSpire; Perkin Elmer). All measurements were done in triplicate and repeated independently at least three times. Identically treated PBS-coated wells were used as blanks.

\section{Statistical analysis}

All obtained values are reported as mean \pm standard deviation. All statistical analyses were performed with IBM SPSS Statistics 20 (IBM). Normal distribution of the data was analyzed by the Kolmogorov-Smirnov test. Student's $t$-test for two independent samples and one-way ANOVA followed by Student-NewmanKeuls (SNK) post hoc test for more than two groups were used to determine differences of indicated groups; $p$-values $<0.05$ were considered significant.

\section{Results}

Establishment of an ex vivo system to study MSC attachment to cartilage lesions

The attachment potential of cells can be analyzed in in vitro cell attachment assays. However, one disadvantage of these in vitro systems is that the coating with specific proteins only partially mimics the complex composition of a specific tissue. Therefore, we developed an ex vivo attachment system in native articular cartilage. To analyze the attachment of hPLAP-tg MSC to defective cartilage in osteochondral explants from wild-type rats, we created a full-thickness defect on the lateral and on the medial compartment of the tibia's articular cartilage, and used the defects as surface for the MSC attachment. To characterize the matrix components exposed in the defects, we performed alcian blue-PAS staining of cryosections.

Figure 1A shows that hyaline cartilage, calcified cartilage, as well as subchondral bone were exposed in the full-thickness defects. Single-cell suspensions of donor MSC isolated from hPLAP-tg rats were allowed to attach to the osteochondral explants. The area covered with hPLAP-tg MSC within the defect was quantified after washing, fixation, and histochemical staining of the hPLAP-tg cells. We first sought to optimize the seeding cell density in this osteochondral explants (Fig. 1B,C). When seeding $10^{4}$ cells per explant, we 

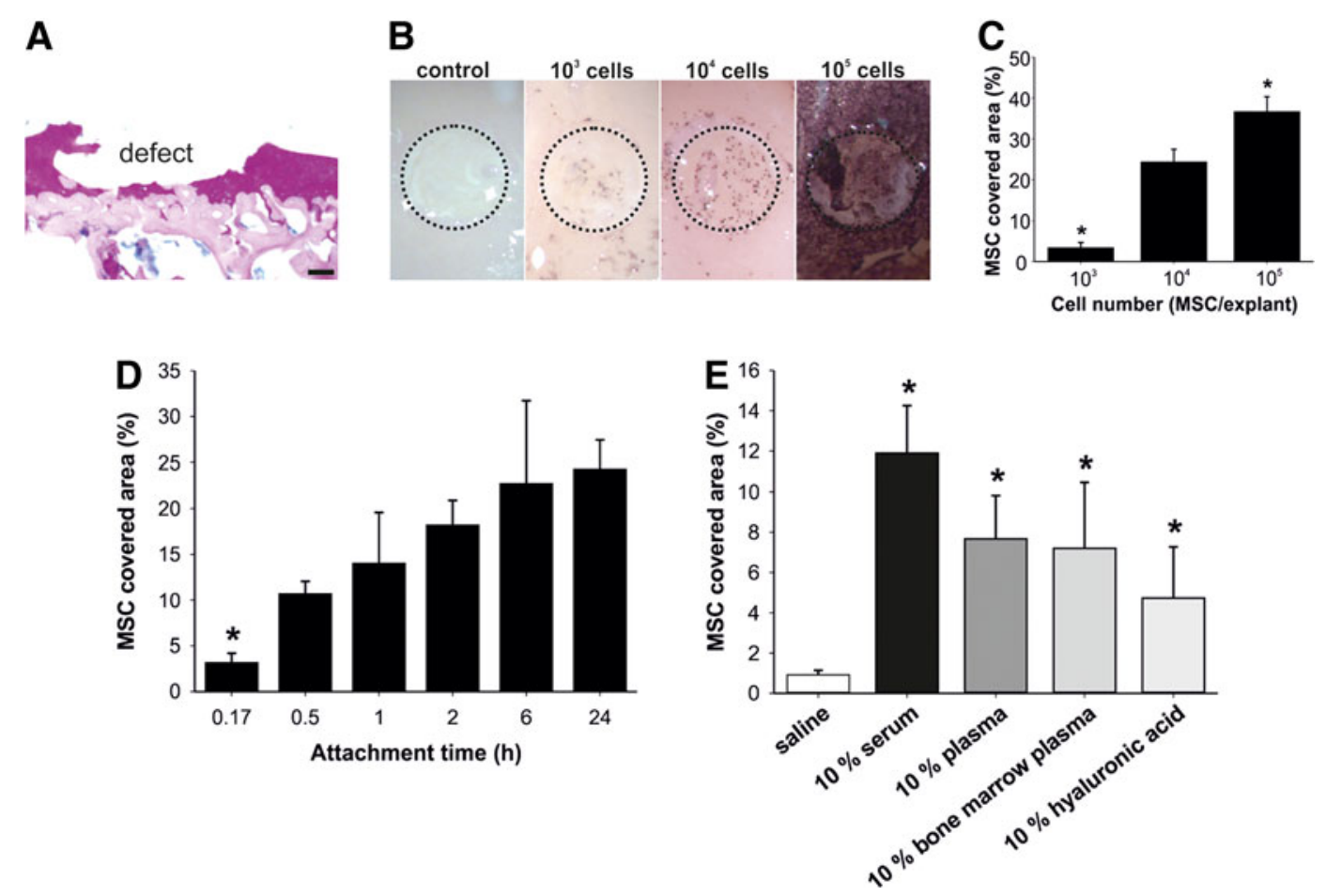

FIG. 1. Establishment of an ex vivo attachment system. (A) Alcian blue-PAS staining of cryosections of osteochondral explants with a full-thickness defect. Articular cartilage is stained purple, subchondral bone is stained magenta, and proteoglycans and nuclei are stained blue. (B, C) Screen for optimal cell number. Ex vivo osteochondral explants from wild-type Fischer 344 rats were used to analyze the attachment of human placental alkaline phosphatase (hPLAP)-tg mesenchymal stem cells (MSC) to cartilage lesions. After attachment, the cells were fixed, and histochemically stained for hPLAP after heat inactivation of endogenous alkaline phosphatases. The stained area within the defect, corresponding to the attached cells, was quantified with the help of ImageJ software. Indicated numbers of cells suspended in $2 \%$ rat serum were allowed to attach for $24 \mathrm{~h}$. Dashed circle in (B) indicates the full-thickness defect on the osteochondral explant. ${ }^{*} p<0.05$ vs. $10^{4}$ cells/ explant by one-way ANOVA followed by Student-Newman-Keuls (SNK) test. (D) Screen for optimal attachment time. An amount of $10^{4} \mathrm{hPLAP}$-tg MSC per explant suspended in $2 \%$ rat serum were allowed to attach for the indicated time to the osteochondral explants. ${ }^{*} p<0.05 \mathrm{vs.} 1 \mathrm{~h}$ incubation time by one-way ANOVA followed by SNK test. (E) Testing different vehicles for MSC attachment to full-thickness cartilage defects. An amount of $10^{4}$ MSC suspended in saline alone or saline supplemented with $10 \%$ rat serum, plasma, bone marrow plasma, or hyaluronic acid were allowed to attach for $55 \mathrm{~min} .{ }^{*} p<0.05 \mathrm{vs}$. saline by one-way ANOVA followed by SNK test. $(\mathbf{F}, \mathbf{G})$ Influence of increasing concentrations of bovine serum to MSC attachment. An amount of $10^{4} \mathrm{MSC}$ suspended in saline supplemented with 0-50\% serum were allowed to attach to osteochondral explants for $55 \mathrm{~min}$. Attachment to intact and defective surfaces was quantified on separate explants. Dashed circle in (F) indicates the full-thickness defect on the osteochondral explant. ${ }^{*} p<0.05$ and ${ }^{* *} p<0.001$ by Student's $t$-test. Data represent mean values $\pm S D(n \geq 3)$. All experiments were performed in triplicate and in at least three independent experiments. (H) Histochemical hPLAP/nuclear fast red staining of osteochondral explants. hPLAP-tg MSC (arrows) were stained in dark violet on cryosections of intact and defective osteochondral explants. Cartilage is stained orange-red and subchondral bone is stained red. Scale bar $=100 \mu \mathrm{m}$. 

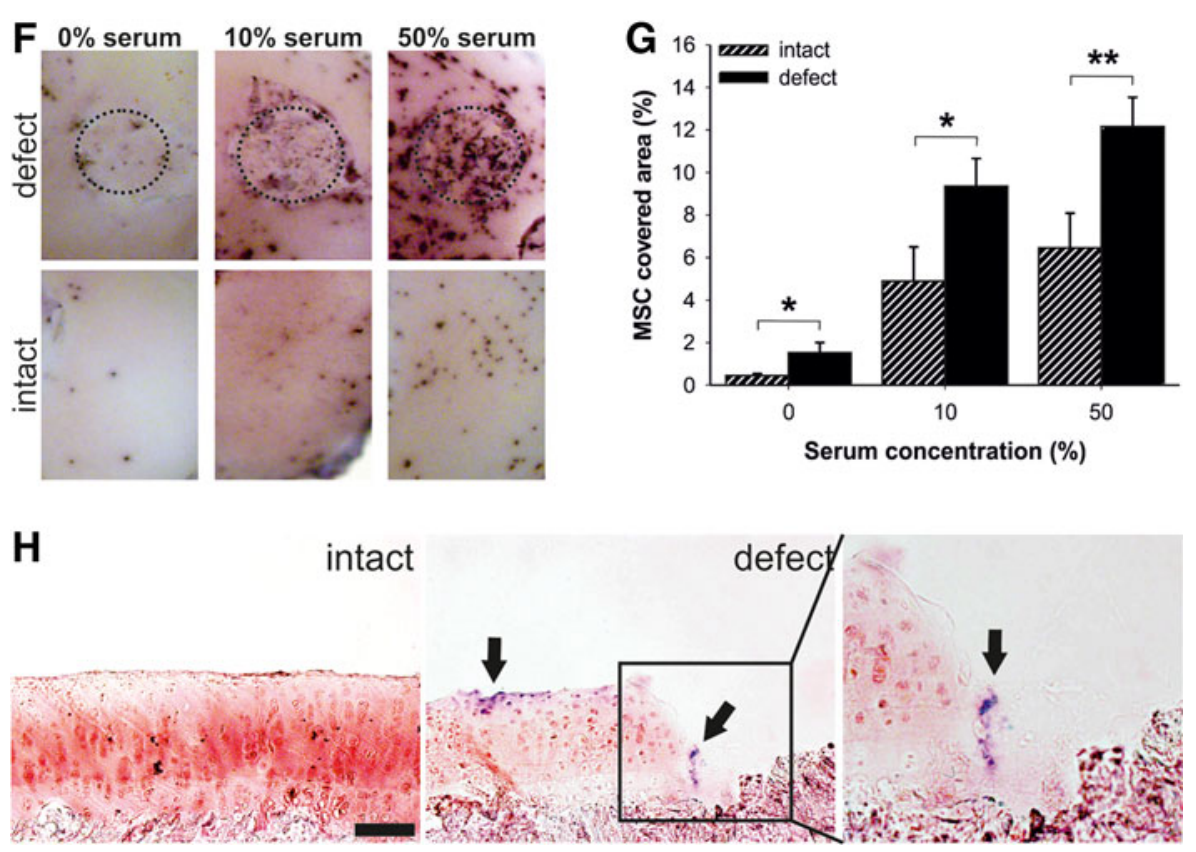

FIG. 1. (Continued).

reached an optimal readout with single cells being distributed homogenously over the defect area, and room for quantification of increases and decreases in cell attachment. The initial attachment of cells is followed by processes such as spreading, proliferation, and/or migration. ${ }^{36}$ Therefore, we next determined the optimal adhesion time for our ex vivo attachment assay. Most of the seeded hPLAP-tg MSC attached within 0.5-1 h to the defective cartilage (Fig. 1D). After $2 \mathrm{~h}$ of incubation, only a small further increase in the covered area could be observed. It is likely that this further increase after prolonged incubation for up to $2 \mathrm{~h}$ was caused by spreading during which the cells get flattened and increase their contact surface. Taken together, we established a novel ex vivo system based on the attachment of hPLAP-tg MSC to cartilage lesions on osteochondral explants. This system is similar to the classic in vitro attachment assay but with the advantage of using native tissue as attachment surface.

Serum and divalent cations facilitate MSC adhesion to defective cartilage

Many studies describe the use of serum as vehicle for MSC application in cell-based therapies. ${ }^{6,10}$ In addition, the use of hyaluronic acid is described to have a positive effect on MSC attachment after intra-articular injection. ${ }^{5,8}$ We therefore tested several vehicles suitable for increasing MSC adhesion to cartilage lesions in our osteochondral explant system. Administration of hPLAP-tg MSC in 10\% rat serum enhanced the attachment compared to saline, as well as $10 \%$ of plasma or bone marrow plasma (Fig. 1E). The use of $10 \%$ hyaluronic acid also increased MSC attachment, but to a lower extent than serum, plasma, or bone marrow plasma. A more detailed analysis showed that the addition of serum dose-dependently increased the MSC-covered area on both the intact and the defective cartilage, but that adhesion to the defective cartilage was significantly higher at all serum concentrations at the used seeding density of $10^{4}$ cells per explant (Fig. $1 F, G)$. For the quantification of MSC attachment on intact explants, independent explants without defect were used. It is interesting to note in Figure $1 \mathrm{~F}$ that on explants with a defect, more MSC attached to the surrounding intact cartilage surface compared to intact explants without a defect. This suggests that already minor damage (also seen in Fig. 1H, middle panel, left arrow) of the cartilage surface in the surroundings of the full-thickness defect induced by handling and cleaning of the sample facilitates attachment of MSC. Histological staining of hPLAP-tg MSC in cryosections 
confirmed the attachment of these cells in and in the surroundings of the full-thickness defects in the osteochondral explants (Fig. 1H). These results show that serum enhances MSC adhesion to cartilage lesions.

Earlier studies reported positive effects of magnesium and calcium on cell attachment. ${ }^{37-39} \mathrm{We}$ therefore tested the ability of divalent cations such as magnesium, calcium, zinc, and manganese to improve the adhesion of MSC to defective cartilage in our system. Magnesium caused a dose-dependent increase in the MSC-covered area up to $10 \mathrm{mM}$ concentrations, whereas calcium and manganese showed only a nonsignificant enhancement at 1 and $2.5 \mathrm{mM}$ (Fig. 2A). Surprisingly, zinc, which is known to have an impact

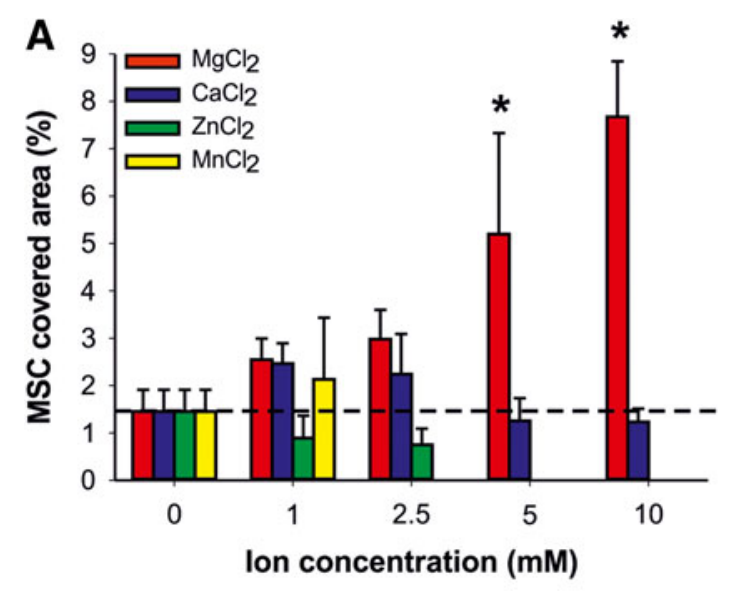

Ion concentration $(\mathrm{mM})$

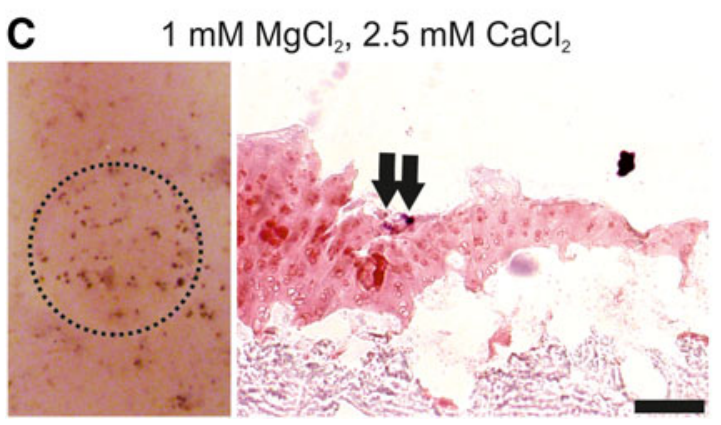

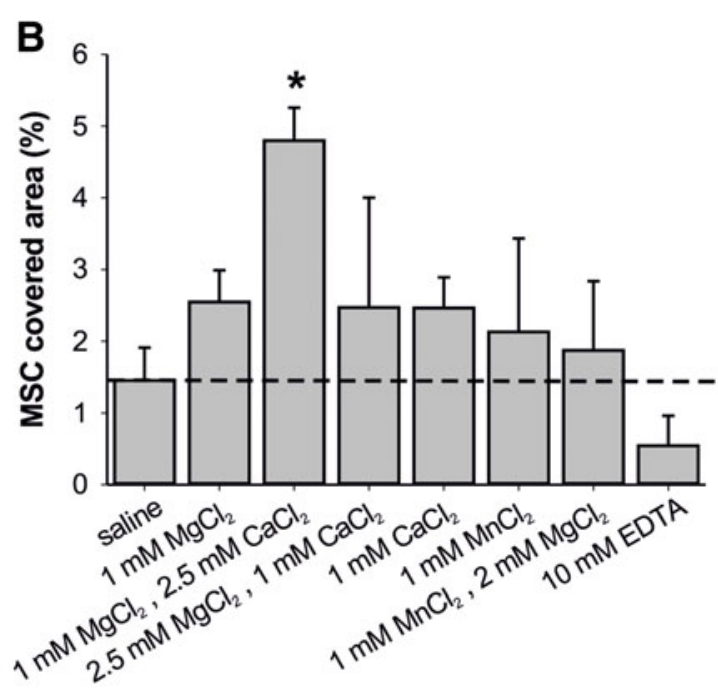

$10 \mathrm{mM}$ EDTA

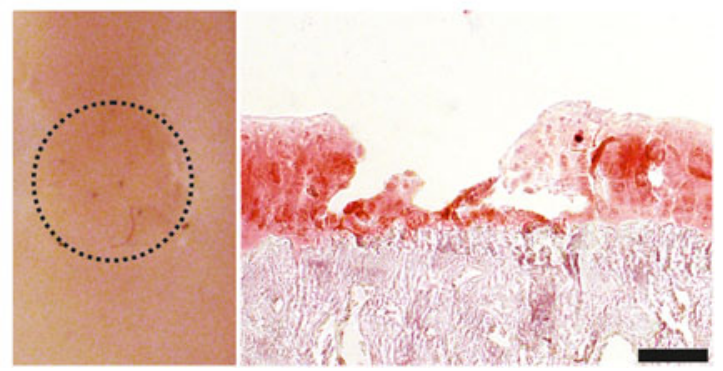

FIG. 2. Divalent cations are necessary for ex vivo MSC attachment to cartilage lesions. (A, B) Influence of different concentrations (A) and combinations (B) of divalent cations on MSC attachment to cartilage defects. MSC suspended in saline were preincubated with the indicated divalent cation concentrations or with $10 \mathrm{mM}$ EDTA, and allowed to attach to osteochondral explants for $55 \mathrm{~min}$. Only attachment to the cartilage defects was quantified. Data represent mean values \pm SD $(n \geq 3)$. All experiments were performed in triplicate and in at least three independent experiments. Note that for $\mathrm{Mn}^{2+}$, only the $1 \mathrm{mM}$ concentration and for $\mathrm{Zn}^{2+}$, only the $1 \mathrm{mM}$ and $2.5 \mathrm{mM}$ concentrations were tested. ${ }^{*} p<0.05$ vs. saline by one-way ANOVA followed by SNK test. (C) Horizontal images and cryosections of hPLAP-stained osteochondral explants seeded with hPLAP-tg MSC in $\mathrm{Mg}^{2+}$ and $\mathrm{Ca}^{2+}$-supplemented saline or EDTA. Left panels show representative horizontal images of hPLAPstained osteochondral explants used for quantification in (B). Dashed circle indicates the full-thickness defect on the osteochondral explant. In hPLAP-stained cryosections, attached hPLAP-tg MSC (arrows) were found in the defects after incubation in saline supplemented with $1 \mathrm{mM} \mathrm{Mg}^{2+}$ and $2.5 \mathrm{mM} \mathrm{Ca}^{2+}$ (left), but not when suspended in $10 \mathrm{mM}$ EDTA (right). Nuclear fast red was used as counterstaining. Cartilage is stained orange-red and subchondral bone is stained red. Scale bar $=100 \mu \mathrm{m}$. 
on the binding affinity and conformation of ECM proteins in cartilage, ${ }^{40}$ had no effect on MSC attachment compared with saline as vehicle. When using combinations of divalent cations as present in MEM medium, a nearly 2-fold increase in MSC adhesion was observed with a near-physiological combination of $1 \mathrm{mM}$ magnesium and $2.5 \mathrm{mM}$ calcium as compared to single components and other ion combinations (Fig. 2B). In addition to saline, the divalent cation chelator EDTA $^{21}$ was used as a negative control. Horizontal images of hPLAP-stained explants and histological staining of attached MSC confirmed these findings (Fig. 2C). In conclusion, we found that serum and the divalent cations magnesium and calcium improved the adhesion of MSC to full-thickness cartilage defects in our explant system.

\section{Collagen I, collagen II, and fibronectin mediate MSC} attachment in vitro

To evaluate the specific matrix components mediating MSC attachment to full-thickness cartilage lesions, we performed in vitro cell attachment assays with prominent ECM proteins of cartilage and bone. In a onepoint measurement to screen for putative interaction partners, we used the following ECM proteins: collagen I, present in bone, and the major cartilage proteins collagen II, collagen XXII, COMP, and fibronectin, which is known to be expressed in bone and cartilage. Only collagen I, collagen II, and fibronectin mediated MSC attachment. MSC adhered neither to collagen XXII, a prominent marker of the articular cartilage surface, ${ }^{31}$ nor to COMP (Fig. 3A). Collagen I, collagen II, and fibronectin mediated the MSC attachment in a concentration-dependent and saturable manner in the presence of the divalent cations manganese and magnesium (Fig. 3B). MSC adhesion to collagen I, collagen II, and fibronectin was inhibited completely by $10 \mathrm{mM}$ EDTA (Fig. 3B).

To compare ex vivo and in vitro experiments, we tested several divalent cations for their ability to influence MSC attachment in vitro. In general, all tested cations had their strongest positive influence on MSC adhesion at physiological concentrations of 1 or $2.5 \mathrm{mM}$. Zinc of $1 \mathrm{mM}$ had the most pronounced effect on MSC adhesion to collagen II (Fig. 3D). Zinc is present at a concentration of about $1 \mathrm{mM}$ in cartilage and has the ability to change the conformation of ECM proteins such as collagen II, collagen IX, and COMP, and influences their binding ability to each other. ${ }^{40}$ Interestingly, calcium had a negative effect on the ad- hesion to collagen I and no effect on the adhesion to collagen II.

In addition, we tested combinations of divalent cations, similar to the above-mentioned ex vivo experiments. Combinations of $1 \mathrm{mM}$ manganese with $2 \mathrm{mM}$ magnesium and of $2.5 \mathrm{mM}$ magnesium with $1 \mathrm{mM}$ calcium had the most positive effects on MSC attachment to collagen I (Fig. 3E). The adhesion of MSC to collagen II was significantly increased when using $1 \mathrm{mM}$ manganese with $2 \mathrm{mM}$ magnesium, or $2.5 \mathrm{mM}$ magnesium with $1 \mathrm{mM}$ calcium (Fig. 3F). In agreement with the experiments shown in Figures $2 \mathrm{~B}$ and $3 \mathrm{~B}$, the attachment of MSC to collagen I and collagen II was almost completely inhibited in the presence of $10 \mathrm{mM}$ EDTA (Fig. 3E,F). Collectively, these data demonstrate that MSC are able to attach, in a divalent cation-dependent manner, to the ECM proteins collagen I, collagen II, and fibronectin, all present in bone and cartilage tissue.

\section{$\beta 1$ integrins mediate MSC attachment}

in vitro and ex vivo

Because the MSC attachment was almost completely abolished in the presence of EDTA, we hypothesized that integrins may be involved in the adhesion of MSC to matrix proteins exposed in cartilage lesions. MSC are known to express the $\beta 1$ integrin subunit on their cell surface, ${ }^{13,14}$ which is also used as a marker to identify MSC after isolation. Furthermore, they express the subunits $\alpha 1, \alpha 2$, and $\alpha 10$, belonging to the family of collagen-binding integrins, and the subunits $\alpha 5$ and $\alpha \mathrm{V}$ belonging to the RGD-binding integrins. ${ }^{14,15}$ Thus, MSC express both collagen- and RGD-binding integrins.

To identify the integrins involved in MSC adhesion to ECM proteins and cartilage lesions, we performed competition and inhibition assays. We first performed competition experiments with RGD peptide in the in vitro attachment system. Only the MSC attachment to fibronectin could be inhibited by about $60 \%$ by the RGD peptide (Fig. 4A). The attachment to collagen I and collagen II was unaffected by the RGD peptide (Fig. 4A). An RGD control peptide served as a negative control. Blocking assays with a monoclonal $\beta 1$ integrin blocking antibody revealed that the $\beta 1$ integrin subunit was essential for binding of MSC to collagen I and collagen II. If the $\beta 1$ integrin subunit was blocked, MSC could no longer adhere to collagen I and collagen II, whereas addition of an isotype control antibody had no influence on the attachment of MSC (Fig. 4B). In contrast, attachment of MSC to fibronectin was not 
A
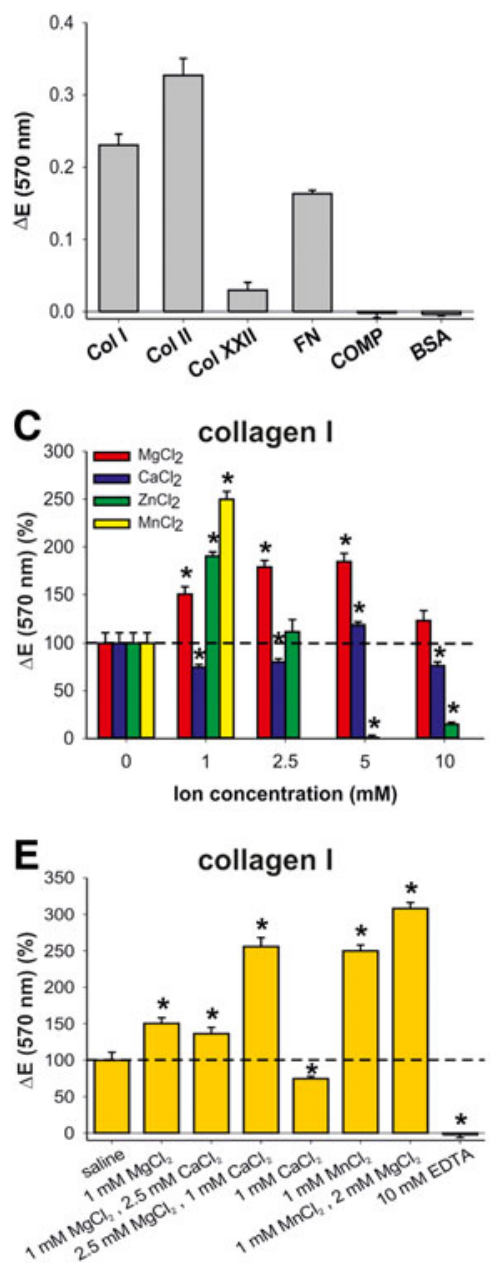
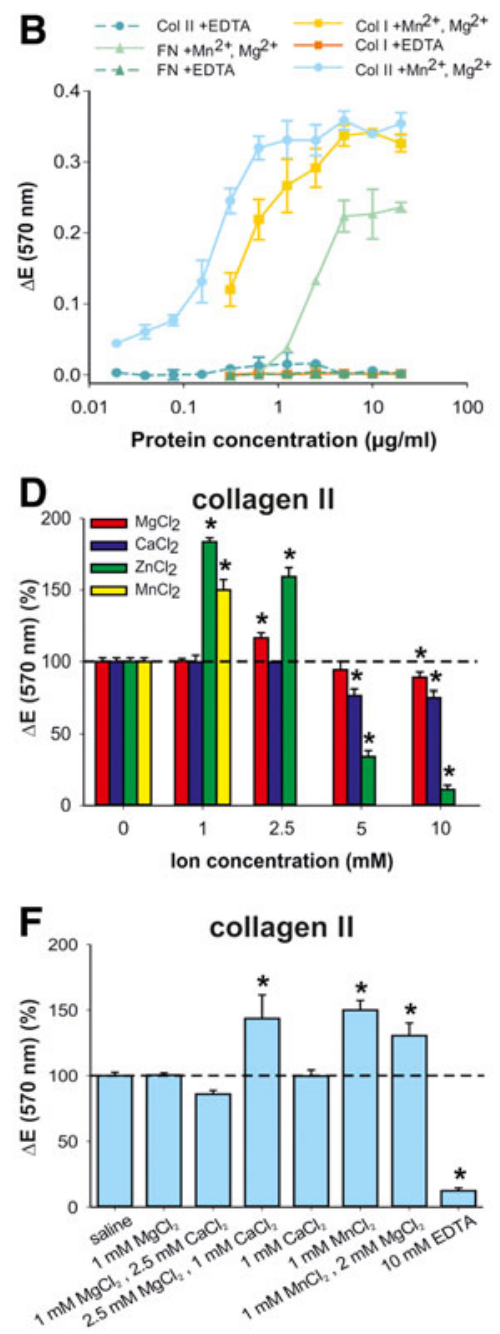

FIG. 3. In vitro attachment of MSC to extracellular matrix (ECM) proteins of bone and cartilage. (A) Screen of MSC adhesion to abundant ECM proteins. An amount of $20 \mu \mathrm{g} / \mathrm{mL}$ of protein was immobilized at $4^{\circ} \mathrm{C}$ overnight.

(B) Adhesion of MSC to collagen I, collagen II, and fibronectin. Serial dilutions of collagen I (Col I, $0.3-20 \mu \mathrm{g} / \mathrm{mL}$ ), collagen II (Col II, 0.02-20 $\mu \mathrm{g} / \mathrm{mL}$ ), and fibronectin (FN, $0.3-20 \mu \mathrm{g} / \mathrm{mL}$ ) were immobilized at $4^{\circ} \mathrm{C}$ overnight. In (A) and (B), MSC suspended in MEM were allowed to attach to the substrate for $55 \mathrm{~min}$ in the presence of $\mathrm{Mn}^{2+}$ and $\mathrm{Mg}^{2+}$ or EDTA, followed by crystal violet staining. Relative attachment is presented as $\Delta E$, measured extinction minus nonspecific attachment to PBS-pretreated wells. Data represent mean values \pm SD. $(n=3)$. At least three independent assays were performed for all experiments. Bovine serum albumin (BSA) was used as negative control. (C, D) Influence of different concentrations of divalent cations on MSC attachment to collagen I and II. An amount of $2.5 \mu \mathrm{g} / \mathrm{mL}$ collagen I (C) or collagen II (D) was immobilized onto 96-well plates overnight. (E, F) Influence of combinations of divalent cations on the attachment of MSC. An amount of $2.5 \mu \mathrm{g} / \mathrm{mL}$ collagen I (E) or collagen II (F) was immobilized overnight. In (C)-(F), MSC suspended in saline were preincubated with the indicated divalent cation concentrations or with $10 \mathrm{mM}$ EDTA, and were allowed to attach to the substrate for $55 \mathrm{~min}$. Relative attachment is presented as relative $\Delta E$, normalized to the attachment $(\Delta E)$ of MSC suspended in saline. Data are mean $\pm \mathrm{SD}(n=3)$. At least three independent assays were performed for all experiments. Note that for $\mathrm{Mn}^{2+}$, only the $1 \mathrm{mM}$ concentration was measured. ${ }^{*} p<0.05$ vs. saline by one-way ANOVA followed by SNK test. 

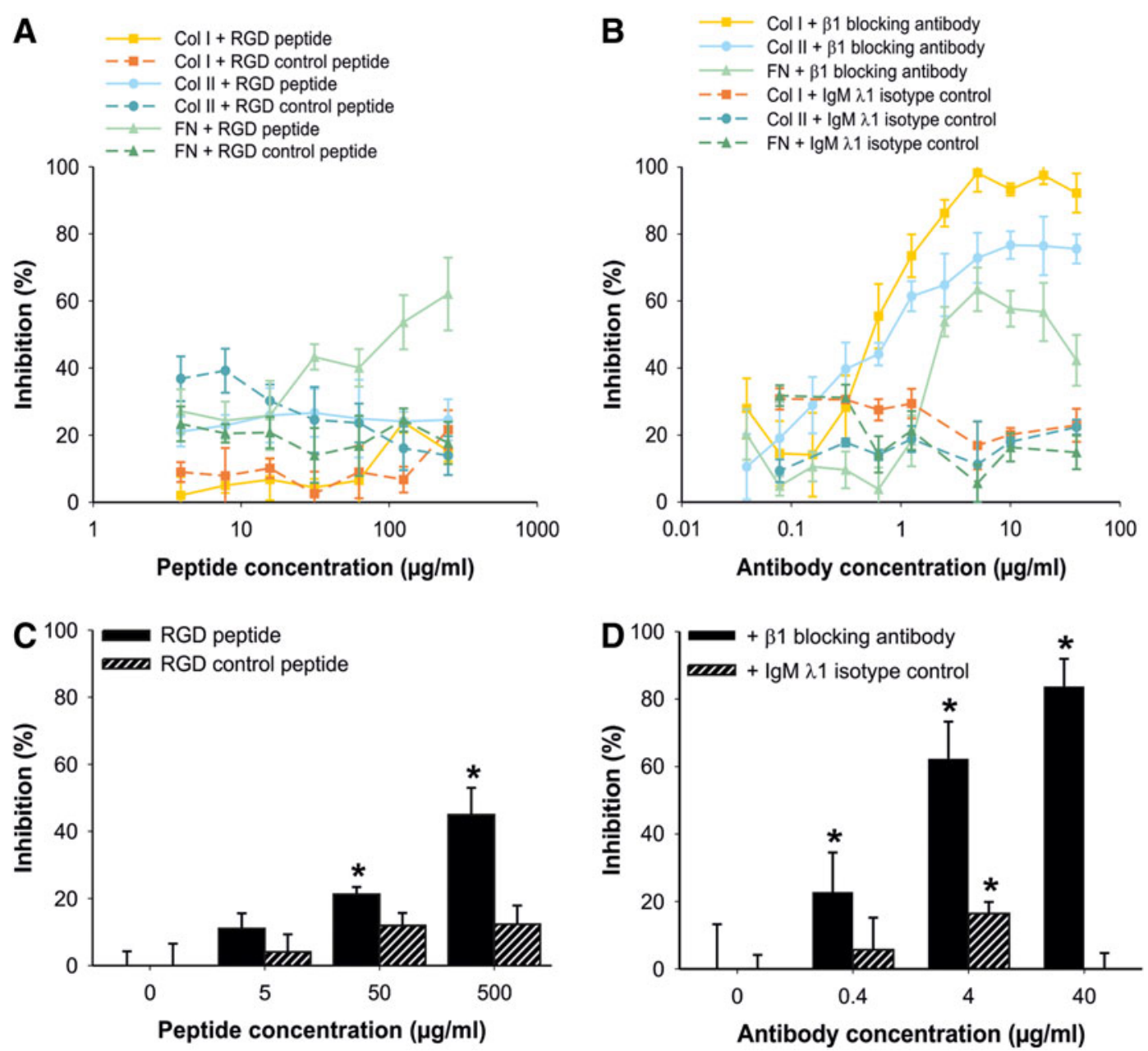

FIG. 4. Adhesion of MSC in vitro and ex vivo is $\beta 1$ integrin dependent. (A, B) Influence of RGD peptides and a $\beta 1$ integrin blocking antibody on MSC attachment to ECM proteins in vitro. An amount of $2.5 \mu \mathrm{g} / \mathrm{mL}$ of collagen I (Col I), collagen II (Col II), and fibronectin (FN) was immobilized at $4^{\circ} \mathrm{C}$ overnight. MSC were suspended in MEM containing $\mathrm{Mn}^{2+}$ and $\mathrm{Mg}^{2+}$, supplemented with the indicated concentrations of RGD peptide, RGD control peptide, $\beta 1$ integrin blocking antibody, or IgM $\lambda 1$ isotype control, and were allowed to attach for 55 min to the substrate. Attachment was quantified as $\Delta E$, measured extinction minus nonspecific attachment to PBSpretreated wells, and is given as \% inhibition. Data represent mean values $\pm \operatorname{SD}(n=3)$. At least three independent assays were performed for all experiments. (C, D) Influence of RGD peptides and a $\beta 1$ integrin blocking antibody on MSC attachment to full-thickness cartilage lesions ex vivo. MSC were suspended in saline containing $50 \%$ bovine serum and supplemented with the indicated concentrations of RGD peptide, RGD control peptide, $\beta 1$ integrin blocking antibody, or $\lg M \lambda 1$ isotype control. MSC were allowed to attach for $55 \mathrm{~min}$ to the osteochondral explants. Only attachment to the cartilage defects was quantified. Data represent mean values $\pm \mathrm{SD}(n \geq 3)$. All experiments were performed in triplicate and in at least three independent experiments. ${ }^{*} p<0.05$ vs. $50 \%$ bovine serum without blocking supplement by one-way ANOVA followed by SNK test. 
completely blocked by the $\beta 1$ integrin blocking antibody (Fig. 4B). These results demonstrate that MSC bind to fibronectin via RGD-binding integrins, and to collagen I and collagen II via collagen-binding integrins.

To confirm the role of RGD- and collagen-binding integrins in MSC attachment to full-thickness cartilage lesions in the more complex ex vivo situation, we performed competition and blocking studies in the osteochondral explant system. The RGD peptide was able to compete for about $50 \%$ of the attachment of MSC to full-thickness cartilage lesions, whereas the RGD control peptide had no influence (Fig. 4C). Blocking studies with a $\beta 1$ integrin blocking antibody demonstrated a dose-dependent inhibition of the MSC attachment to defective cartilage with a maximum inhibition of about $90 \%$ at $40 \mu \mathrm{g} / \mathrm{mL}$ antibody concentration, whereas an isotype control antibody had no influence on MSC attachment (Fig. 4D). These results fully corroborate our in vitro findings and clearly show that attachment of MSC to full-thickness cartilage lesions in our ex vivo system is almost entirely $\beta 1$ integrin-mediated, whereby both RGD- and collagen-binding integrins are involved.

To identify potential integrin $\alpha$-subunits involved in the adhesion of MSC to full-thickness cartilage defects, we performed immunofluorescence analyses (Fig. 5). Staining of cryosections of intact osteochondral explants for collagen I and II confirmed the specificity of the used antibodies. The anti-collagen I antibody clearly stained the bone tissue, whereas collagen II was expressed exclusively in the articular cartilage and calcified cartilage of the epiphysis in intact osteochondral explants (Fig. 5A). The collagen I and II staining pattern was similar to the distribution of bone and cartilage tissue as evidenced by alcian blue-PAS staining (Fig. 5B). Co-immunofluorescence analysis for $\alpha 2$ integrin and collagen I and II demonstrated distinct zones of adjacent expression of $\alpha 2$ integrin and collagen I and II in areas where MSC attached to subchondral bone or hyaline cartilage, respectively (Fig. 5C and D). In contrast, expression of $\alpha 1$ (Fig. 5E) or $\alpha 5$ integrin (Fig. 5F) was not observed in the defects. Taken together, our findings suggest that $\alpha 2 \beta 1$ integrin may be one of the key mediators of MSC attachment to bone and cartilage in full-thickness cartilage lesions.

\section{Discussion}

The key findings in our study were (1) that serum improves MSC attachment to cartilage lesions, (2) that divalent cations are essential for binding of MSC to full-thickness cartilage lesions as well as for MSC bind- ing to collagen I, collagen II, and fibronectin, (3) that $\beta 1$ integrin blocking antibodies fully block binding of rat MSC to collagen I and collagen II, but only partially inhibit binding to fibronectin, and (4) that RGD peptides partially block the adhesion of MSC to fibronectin in vitro and to cartilage lesions ex vivo. Thus, our data suggest that both RGD- and collagen-binding integrins are involved in the attachment of MSC to full-thickness articular cartilage lesions. This model is shown in Figure 6. Our study is in line with the findings of Shimaya and co-workers ${ }^{37}$ that magnesium increased adhesion of human synovial MSC to collagen-coated slides in a $\beta 1$ integrin-dependent fashion in vitro, underscoring the importance of $\beta 1$ integrins for binding of MSC to ECM proteins across species.

The present study has shown that our newly established co-isogeneic ex vivo system consisting of MSC isolated from hPLAP-tg donor F344 rats and wildtype F344 osteochondral explants is an excellent tool to study MSC attachment to cartilage lesions. By use of the marker gene $h P L A P$, which is ubiquitously and stably expressed in hPLAP-tg rats and does not influence MSC behavior, ${ }^{26,27}$ we were able to reliably and very efficiently track hPLAP-expressing MSC attached to the wild-type osteochondral explants by histochemical detection methods and subsequent image analysis.

In our ex vivo explant system, the near-physiological combination of $1 \mathrm{mM} \mathrm{MgCl} 2$ with $2.5 \mathrm{mM} \mathrm{CaCl}_{2}$ provided the best results in terms of MSC-covered area within the cartilage lesion. Interestingly, calcium had no or even a negative effect on the adhesion of MSC to defective cartilage. Conversely, adhesion of MSC to collagen I, collagen II, and fibronectin in vitro was abolished in the presence of $10 \mathrm{mM}$ EDTA. These phenomena have already been described earlier ${ }^{38,39}$ and are consistent with the notion that integrins may be involved in the MSC attachment. Divalent cations are needed for activation of integrins and for keeping them in their active state. ${ }^{19-21}$ ETDA chelates divalent cations, resulting in an inactive state of cell surface integrins. ${ }^{21}$ Thus, our data suggest that the attachment of MSC to ECM proteins accessible in full-thickness cartilage defects is an almost entirely integrin-mediated process.

Cultured MSC express various integrin subunits such as $\alpha 1, \alpha 2, \alpha 3, \alpha 4, \alpha 5, \alpha 6, \alpha 7, \alpha 10, \alpha \mathrm{V}, \alpha \mathrm{IIb}, \beta 1$, $\beta 3, \beta 4$, and $\beta 5$. $^{13-15}$ The expression of these integrin subunits on MSC was described earlier on mRNA and protein level. Expression of the subunits $\alpha 7, \alpha 10$, $\alpha \mathrm{IIb}$, and $\beta 5$ has so far only been analyzed by microarray hybridization. ${ }^{15}$ Our in vitro cell attachment assays 

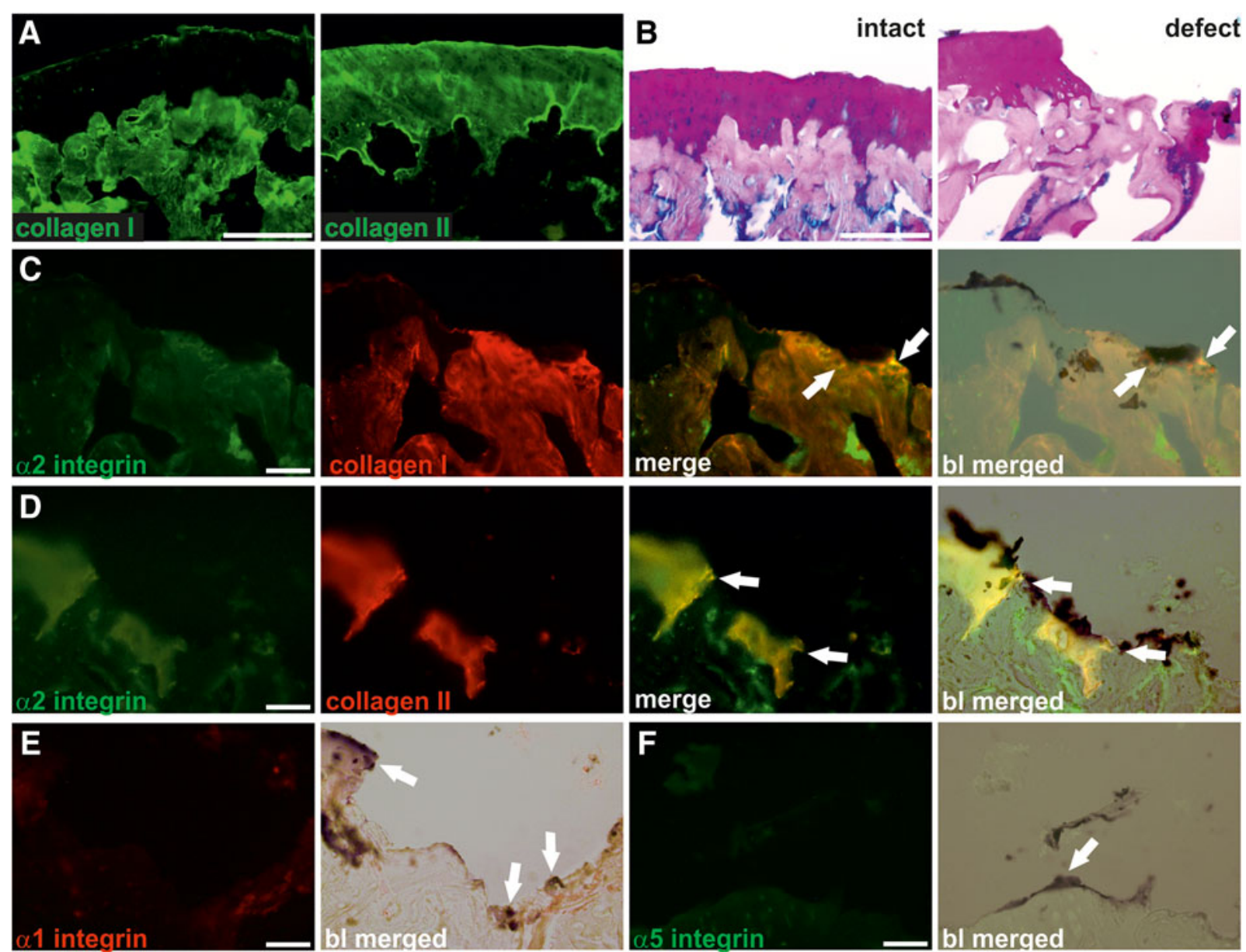

FIG. 5. Expression of $\alpha 2$ integrin at MSC attachment sites to bone and cartilage. (A) Expression of collagen I and collagen II in cryosections of intact osteochondral explants. Immunofluorescence analysis was performed on cryosections of hPLAP prestained intact osteochondral explants by incubation with polyclonal antibodies against collagen I and collagen II. Collagen I is found in bone tissue, whereas collagen II is exclusively located in cartilage tissue of the intact explant. (B) Alcian blue-PAS staining of intact osteochondral explants and explants with a full-thickness defect. Articular cartilage is stained purple, subchondral bone is stained magenta, and proteoglycans are stained blue. (C, D) Expression of $\alpha 2$ integrin in osteochondral explants with full-thickness defects. Co-immunofluorescence analysis was performed on cryosections of previously hPLAP-stained and quantified osteochondral explants by parallel incubation with an antibody against $\alpha 2$ integrin (C, D, green), and a polyclonal antibody against collagen I (C, red) and collagen II (D, red). $\alpha 2$ integrin and collagen I and II are co-expressed in distinct adjacent zones (merge, yellow, arrows). Merging with bright light (bl) images demonstrated the co-expression of $\alpha 2$ integrin and collagen I at sites where hPLAP-tg MSC attach to subchondral bone ( $C$, bl merged, arrows). Bright light-merged images after co-staining of $\alpha 2$ integrin and collagen II revealed co-expressing sites where hPLAP-tg MSC attach to collagen II (D, bl merged, arrows). (E, F) Expression of $\alpha 1$ and $\alpha 5$ integrin in osteochondral explants with full-thickness defects. Immunofluorescence analysis was performed on hPLAP-prestained cryosections of osteochondral explants by incubation with a monoclonal antibody against $\alpha 1$ integrin (E) and a polyclonal anti- $\alpha 5$ integrin antibody (F). Expression of neither $\alpha 1$ nor $\alpha 5$ integrin could be observed at sites where hPLAP-tg MSC attach to the cartilage lesion (bl merged, arrows). Scale bar $=50 \mu \mathrm{m}$. 


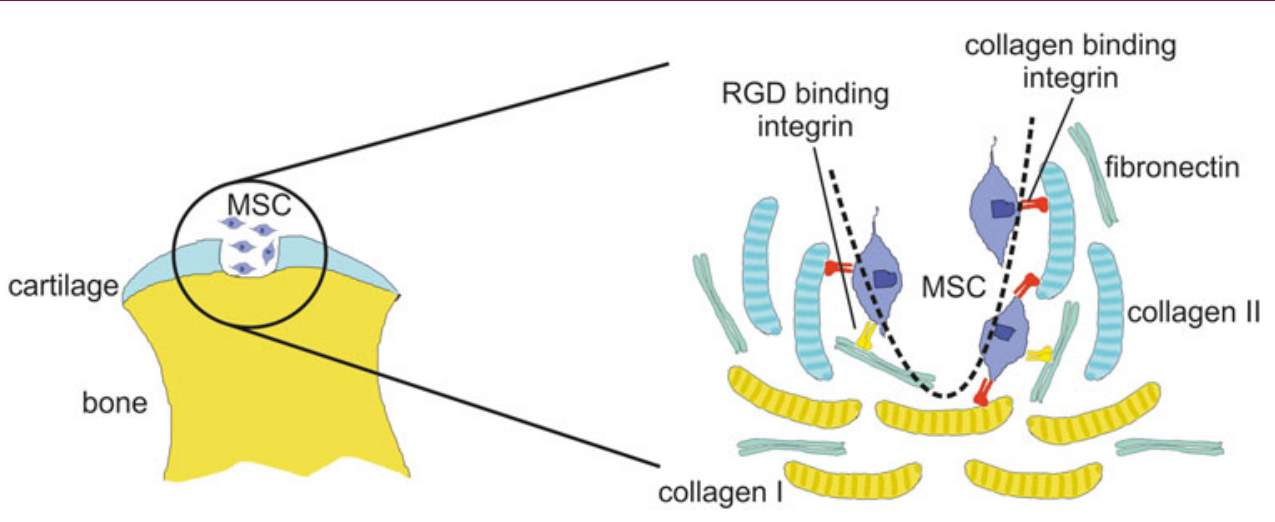

FIG. 6. Model for MSC adhesion to articular cartilage lesions. MSC are able to bind to collagen I in bone and to collagen II in cartilage in full-thickness cartilage defects (dashed line) via collagen-binding $\beta 1$ integrins. In addition, MSC interact via RGD-binding integrins with fibronectin, a major matrix protein present in bone and cartilage.

revealed that MSC attach to collagen I, collagen II, and fibronectin, but not to collagen XXII and COMP. Collagen I is the main ECM protein in bone, collagen II is the major matrix molecule in cartilage, and fibronectin is present in high amounts in both tissues. In agreement with the fact that all collagen-binding integrins $(\alpha 1 \beta 1, \alpha 2 \beta 1, \alpha 10 \beta 1$, and $\alpha 11 \beta 1)$ contain the $\beta 1$-subunit, ${ }^{20}$ binding of MSC to collagen I and collagen II in vitro was fully blocked by the monoclonal $\beta 1$ integrin blocking antibody. Furthermore, immunofluorescence analysis on osteochondral explants with cartilage lesions demonstrated expression of $\alpha 2$ integrin in distinct zones where MSC attach to collagen I or collagen II in the lesion, whereas no expression of $\alpha 1$ and $\alpha 5$ integrin could be observed. These distinct zones are likely to be focal adhesion sites that can develop into focal adhesion plaques, and are known as first step of integrin signaling. ${ }^{20}$

The finding that $\alpha 2 \beta 1$ integrin may be involved in MSC attachment to cartilage lesions explains the strong influence of magnesium and the lacking influence of calcium in our ex vivo attachment experiments, because $\alpha 2$ integrin needs the presence of magnesium rather than calcium to bind to collagens. ${ }^{41,42}$ In addition, $\beta 1$ integrins were also shown to be essential for MSC attachment and survival in biodegradable polymers. ${ }^{18}$ In contrast to collagens, fibronectin interacts with RGD-binding integrins (e.g., $\alpha 5 \beta 1, \alpha \mathrm{V} \beta 1, \alpha \mathrm{V} \beta 3$, $\alpha \mathrm{V} \beta 5$, and $\alpha \operatorname{IIb} \beta 3){ }^{20}$ It is interesting to note in this context that the attachment of MSC to fibronectin could not be fully inhibited by the $\beta 1$ integrin blocking antibody in our experiments, a finding which may indicate that additional RGD-binding $\beta$-subunits are involved in the attachment to fibronectin. Because we were unable to detect expression of $\alpha 5$ integrin in immunofluorescence analysis, it is tempting to speculate that $\alpha \mathrm{V}$ integrins may mediate the attachment of MSC to fibronectin. Clearly, more work needs to be done to fully characterize all integrins involved in the attachment of MSC to cartilage lesions.

MSC for cell-based therapies are usually suspended in saline, ${ }^{7}$ hyaluronic acid, ${ }^{5,8}$ or serum ${ }^{6,10}$ prior to injection. Our study demonstrated that the addition of serum to the cell suspension provided the best results in terms of MSC attachment to full-thickness cartilage lesions, when compared with plasma, bone marrow plasma, hyaluronic acid, or saline. The difference between serum and plasma might be explained by the fact that EDTA was used to prevent blood coagulation during harvesting of plasma samples. Serum is composed of numerous components, and it is currently unknown which specific serum proteins are able to modulate ex vivo and in vivo MSC attachment. Clearly, more work has to be done to characterize the individual factors present in serum that improve MSC attachment to cartilage lesions. It is obvious that this knowledge could be exploited to further refine MSCbased therapies.

In conclusion, our study demonstrates the crucial function of $\beta 1$ integrins for the attachment of MSC to ECM proteins accessible to binding in full-thickness cartilage defects. More experimentation is required to 
exactly define the nature of the individual $\alpha$-subunits, and possibly additional $\beta$-subunits involved in the attachment process of MSC to ECM proteins present in bone and cartilage. A better understanding of the molecular mechanisms involved in MSC adhesion may lead to significant improvements in MSC-based therapies for osteochondral defect regeneration.

\section{Acknowledgments}

We thank Anita Smolnik for help with the histological procedures; Manuel Koch, University of Cologne, for providing recombinant collagen XXII protein; Katrin Blumbach and Beate Eckes for great help with integrin antibodies; and Mats Paulsson, University of Cologne, for intellectual input. The study was supported by a grant from the Austrian Science Fund (FWF P21903) to R.G.E. M.F. was supported by a travel grant from the European Science Foundation Networking Programme REMEDIC. F.Z. was supported by a grant from the Deutsche Forschungsgemeinschaft (DFG ZA $561 / 2-1)$.

\section{Author Disclosure Statement}

\section{No competing financial interests exist.}

\section{References}

1. Prockop DJ, Gregory CA, Spees JL. One strategy for cell and gene therapy: harnessing the power of adult stem cells to repair tissues. Proc Natl Acad Sci USA. 2003;100:11917-11923.

2. Felson DT, Lawrence RC, Dieppe PA, et al. Osteoarthritis: new insights. Part 1: the disease and its risk factors. Ann Intern Med. 2000;133:635-646.

3. Ulrich-Vinther M, Maloney MD, Schwarz EM, et al. Articular cartilage biology. J Am Acad Orthop Surg. 2003;11:421-430.

4. Felson DT, Lawrence RC, Hochberg MC, et al. Osteoarthritis: new insights Part 2: treatment approaches. Ann Intern Med. 2000;133:726-737.

5. Murphy JM, Fink DJ, Hunziker EB, et al. Stem cell therapy in a caprine model of osteoarthritis. Arthritis Rheum. 2003;48:3464-3474.

6. F. Davatchi, Abdollahi BS, Mohyeddin M, et al. Mesenchymal stem cell therapy for knee osteoarthritis. Preliminary report of four patients. Int J Rheum Dis. 2011;14:211-215.

7. Agung $M$, Ochi $M$, Yanada $S$, et al. Mobilization of bone marrow-derived mesenchymal stem cells into the injured tissues after intraarticular injection and their contribution to tissue regeneration. Knee Surg Sports Traumatol Arthrosc. 2006;14:1307-1314.

8. Lee $\mathrm{KB}$, Hui JH, Song IC, et al. Injectable mesenchymal stem cell therapy for large cartilage defects-a porcine model. Stem Cells. 2007;25:29642971.

9. Wakitani S, Goto T, Pineda SJ, et al. Mesenchymal cell-based repair of large, full-thickness defects of articular cartilage. J Bone Joint Surg Am. 1994;76:579-592.

10. Wakitani S, Imoto K, Yamamoto T, et al. Human autologous culture expanded bone marrow mesenchymal cell transplantation for repair of cartilage defects in osteoarthritic knees. Osteoarthritis Cartilage. 2002;10:199-206.

11. Emadedin M, Aghdami N, Taghiyar L, et al. Intra-articular injection of autologous mesenchymal stem cells in six patients with knee osteoarthritis. Arch Iran Med. 2012;15:422-428.

12. Bianco $P$, Cao $X$, Frenette PS, et al. The meaning, the sense and the significance: translating the science of mesenchymal stem cells into medicine. Nat Med. 2013;19:35-42.
13. Pittenger MF, Mackay AM, Beck SC, et al. Multilineage potential of adult human mesenchymal stem cells. Science 1999;284:143-147.

14. Majumdar MK, Keane-Moore M, Buyaner D, et al. Characterization and functionality of cell surface molecules on human mesenchymal stem cells. J Biomed Sci. 2003;10:228-241.

15. Goessler UR, Bieback K, Bugert $\mathrm{P}$, et al. In vitro analysis of integrin expression during chondrogenic differentiation of mesenchymal stem cells and chondrocytes upon dedifferentiation in cell culture. Int J Mol Med. 2006;17:301-307.

16. Connelly JT, Petrie TA, Garcia AJ, et al. Fibronectin- and collagen-mimetic ligands regulate bone marrow stromal cell chondrogenesis in threedimensional hydrogels. Eur Cells Mater. 2011;22:168-176; discussion 176-167.

17. Lam J, Segura T. The modulation of MSC integrin expression by RGD presentation. Biomaterials 2013;34:3938-3947.

18. Lee JW, Kim YH, Park KD, et al. Importance of integrin beta1-mediated cell adhesion on biodegradable polymers under serum depletion in mesenchymal stem cells and chondrocytes. Biomaterials 2004;25:1901-1909.

19. Arnaout MA, Mahalingam B, Xiong JP. Integrin structure, allostery, and bidirectional signaling. Annu Rev Cell Dev Biol. 2005;21:381-410.

20. Hynes RO. Integrins: bidirectional, allosteric signaling machines. Cell. 2002;110:673-687.

21. Springer TA. Complement and the multifaceted functions of VWA and integrin I domains. Structure 2006;14:1611-1616.

22. Humphries JD, Byron A, Humphries MJ. Integrin ligands at a glance. J Cell Sci. 2006;119:3901-3903.

23. Heinegard D, Oldberg A. Structure and biology of cartilage and bone matrix noncollagenous macromolecules. FASEB J. 1989;3:2042-2051.

24. Mendler M, Eich-Bender SG, Vaughan L, et al. Cartilage contains mixed fibrils of collagen types II, IX, and XI. J Cell Biol. 1989;108:191-197.

25. Budde $B$, Blumbach $\mathrm{K}$, Ylostalo $\mathrm{J}$, et al. Altered integration of matrilin-3 into cartilage extracellular matrix in the absence of collagen IX. Mol Cell Biol. 2005;25:10465-10478.

26. Unger NJ, Odorfer Kl, Weber $\mathrm{K}$, et al. Utility of human placental alkaline phosphatase as a genetic marker for cell tracking in bone and cartilage. Histochem Cell Biol. 2007;127:669-674.

27. Balmayor ER, Flicker M, Kaser T, et al. Human placental alkaline phosphatase as a tracking marker for bone marrow mesenchymal stem cells. BioRes Open Access. 2013;2:346-355.

28. Kisseberth WC, Brettingen NT, Lohse JK, et al. Ubiquitous expression of marker transgenes in mice and rats. Dev Biol. 1999;214:128-138.

29. Odorfer KI, Unger NJ, Weber K, et al. Marker tolerant, immunocompetent animals as a new tool for regenerative medicine and long-term cell tracking. BMC Biotechnol. 2007;7:30.

30. Miao D, Scutt A. Histochemical localization of alkaline phosphatase activity in decalcified bone and cartilage. J Histochem Cytochem. 2002;50:333-340.

31. Koch M, Schulze J, Hansen U, et al. A novel marker of tissue junctions, collagen XXII. J Biol Chem. 2004;279:22514-22521.

32. Zwolanek D, Veit G, Eble JA, et al. Collagen XXII binds to collagen-binding integrins via the novel motifs GLQGER and GFKGER. Biochem J. 2014;459:217-227.

33. Eble JA, Beermann B, Hinz $\mathrm{HJ}$, et al. Alpha 2 beta 1 integrin is not recognized by rhodocytin but is the specific, high affinity target of rhodocetin, an RGD-independent disintegrin and potent inhibitor of cell adhesion to collagen. J Biol Chem. 2001;276:12274-12284.

34. Dodge GR, Poole AR. Immunohistochemical detection and immunochemical analysis of type II collagen degradation in human normal, rheumatoid, and osteoarthritic articular cartilages and in explants of bovine articular cartilage cultured with interleukin 1. J Clin Invest. 1989;83:647-661.

35. Hansen U, Platz N, Becker A, et al. A secreted variant of cartilage oligomeric matrix protein carrying a chondrodysplasia-causing mutation (p.H587R) disrupts collagen fibrillogenesis. Arthritis Rheum. 2011;63: 159-167.

36. Partridge MA, Marcantonio EE. Initiation of attachment and generation of mature focal adhesions by integrin-containing filopodia in cell spreading. Mol Biol Cell. 2006;17:4237-4248.

37. Shimaya M, Muneta $T$, Ichinose $S$, et al. Magnesium enhances adherence and cartilage formation of synovial mesenchymal stem cells through integrins. Osteoarthritis Cartilage. 2010;18:1300-1309. 
38. Grzesiak JJ, Davis GE, Kirchhofer D, et al. Regulation of alpha 2 beta 1-mediated fibroblast migration on type I collagen by shifts in the concentrations of extracellular Mg2 + and Ca2 + . J Cell Biol. 1992;117: 1109-1117.

39. Kirchhofer D, Grzesiak J, Pierschbacher MD. Calcium as a potential physiological regulator of integrin-mediated cell adhesion. J Biol Chem. 1991;266:4471-4477

40. Rosenberg $\mathrm{K}$, Olsson $\mathrm{H}$, Morgelin $\mathrm{M}$, et al. Cartilage oligomeric matrix protein shows high affinity zinc-dependent interaction with triple helical collagen. J Biol Chem. 1998;273:20397-20403.

41. Calderwood DA, Tuckwell DS, Eble J, et al. The integrin alpha1 A-domain is a ligand binding site for collagens and laminin. J Biol Chem. 1997:272:12311-12317.

42. Tuckwell D, Calderwood DA, Green LJ, et al. Integrin alpha 2 I-domain is a binding site for collagens. J Cell Sci. 1995;108:1629-1637.

Cite this article as: Zwolanek D, Flicker $M$, Kirstätter $E$, Zaucke $F$, van Osch GJVM, Erben RG (2015) B1 integrins mediate attachment of mesenchymal stem cells to cartilage lesions, BioResearch Open Access 4:1, 39-53, DOI: 10.1089/biores.2014.0055

\section{Abbreviations Used}

$\mathrm{BCIP}=3$-bromo-4-chloro-3-indolyl phosphate $\mathrm{BSA}=$ bovine serum albumin

$\mathrm{COMP}=$ cartilage oligomeric matrix protein

$\mathrm{ECM}=$ extracellular matrix

$F B S=$ fetal bovine serum

$\mathrm{hPLAP}=$ human placental alkaline phosphatase

$M E M=$ minimum essential medium

MSC $=$ mesenchymal stem cells

$\mathrm{NBT}=$ nitrotetrazolium blue chloride

$\mathrm{OA}=$ osteoarthritis

$\mathrm{RT}=$ room temperature

SNK $=$ Student-Newman-Keuls

\section{Publish in BioResearch Open Access}

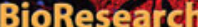

OPEN ACCESS

60

30

A.

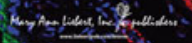

- Broad coverage of biomedical research - Immediate, unrestricted online access

- Rigorous peer review

- Compliance with open access mandates

- Authors retain copyright

- Highly indexed

- Targeted email marketing

liebertpub.com/biores 